\title{
Molecular Genetics and Epigenetics of Ankyloglossia
}

\author{
Lili Nur Indah Sari' ${ }^{1}$, Elza Ibrahim Auerkari² \\ ${ }^{1}$ Pediatric Dentistry Residency Program, Faculty of Dentistry, University of Indonesia, Jakarta \\ 10430, Indonesia \\ ${ }^{2}$ Department of Oral Biology, Faculty of Dentistry, University of Indonesia, Jakarta 10430, \\ Indonesia \\ *Email: elza.ibrahim@ui.ac.id
}

\begin{abstract}
Ankyloglossia is an autosomal dominant condition with in complete penetrence, haracterized by frenulum lingual hypertrophy that produces a thicker and shorter tongue, which may cause significant problems with breastfeeding. It also causes speech and oral motor problems. The prevalence of ankyloglossia is approximately $4 \%$ in babies, and tongue tie is estimated to affect 489,343 newborns, representing $10 \%$ of the Indonesian newborn population of 4,893,435.1 For reasons unknown, this condition is seen more often in males than in females. Ankyloglossia may be isolated or accompanied by cleft palate (CPX). The presentation of this condition is associated with nucleotide base substitution mutations in the TBX22 gene and the G-protein-coupled receptor Lgr5 and can be related to epigenetic modification, including DNA methylation and SUMOylation, which is one kind of histone modification.
\end{abstract}

Keywords: Ankyloglossia, Tongue tie, Genetics, Epigenetics, Lingual frenulum

\section{Introduction}

Ankyloglossia is an autosomal dominant disease with incomplete penetrance that is more common in newborns than in adults. Ankyloglossia is characterized by frenulum lingual hypertrophy, which results in a thicker and shorter tongue that may cause significant problems with breastfeeding; it also causes speech and oral motor problems. Ankyloglossia may be an isolated presentation or accompanied by cleft palate (CPX).

The presentation of ankyloglossia can be associated with gene mutations or epigenetic modifications. Epigenetics is the study of changes in the phenotype or gene expression caused by mechanisms other than changes in the DNA sequence, which affect how the cell reads a gene; this alteration in gene expression is inherited and may be affected by the surrounding environment. A deeper understanding of epigenetic networks will lead to innovations in the treatment and prevention of ankyloglossia.

\section{Ankyloglossia}

\subsection{Definition of ankyloglossia}

The term "Ankyloglossia" was derived from the Greek words "angkilos" (crooked) and "glossa" (tongue). It is also known as "tongue tie" and is a congenital disorder 
commonly found in the newborn population. Ankyloglossia is characterized by hypertrophy of the lingual frenulum, resulting in a tongue that is shorter than usual and with a limited tip [2,3]. The clinical phenotype varies from complete ankyloglossia, a condition in which the ventral portion of the tongue attaches to the base of the mouth, to a clinically minor abnormality [2,3]. Anchor extension between the base of the mouth and the tongue caused by cellular degeneration failure results in the formation of ankyloglossia conditions [3].

Diagnosis of ankyloglossia may be established based on the patient's history and confirmed by characteristic physical examination findings, wherein the frenulum can be thick and fibrous or thin and membranous but is commonly short. The tongue may have a heart shape with a notch when brought forward, due to tethering by the frenulum [4].

\subsection{Prevalence of ankyloglossia}

During growth and development, natural reduction of the lingual frenulum usually occurs between 6 months and 6 years of age [2]. The prevalence of ankyloglossia in babies is approximately $4 \%$ (varying greatly from $0.02 \%$ to $10.7 \%$ ), and tongue tie is estimated to affect 489,343 newborns, representing $10 \%$ of the Indonesian newborn population of $4,893,435.1$ For reasons unknown, this condition is seen more often in males than in females. The male-to-female ratio varies from 1.5:1 to 2.6:1.2,3 Children born to mothers who use cocaine have a 3-fold elevated risk of ankyloglossia [3]. Han et al (2012) reported a transmission rate for ankyloglossia of $20.69 \%$ in 58 cases with hereditary conditions [5].

\subsection{Classification}

The severity of ankyloglossia based on the functional level of the tongue does not yet have a clear and uniform classification [2,3]. Kotlow classified ankyloglossia into four classes based on the ability to lift the tongue (normal range of the free tongue is greater than $16 \mathrm{~mm}$ ) as follows: class IV, complete ankyloglossia (less than $3 \mathrm{~mm}$ ); class III, severe ankyloglossia (3-7 mm); class II, moderate ankyloglossia (8-10 mm); and class 1, mild ankyloglossia (12-16 mm) [6].

\subsection{Potential manifestation}

It is known that a shorter than usual lingual frenulum may result in variable decreased movement of the tongue. Ankyloglossia may lead to a host of problems, such as difficulty with breast feeding. Problems previously reported by mothers of children with ankyloglossia include sore nipples, poor latching and sucking mechanism, low baby weight, hypernatraemic dehydration, and faster weaning [4,7]. In addition, problems with swallowing or dentition, abnormal oral motor function, and various social problems related to the limited movement and function of the tongue can occur. The inability to raise the tip of the tongue toward the upper teeth or upper lip is the major problem faced by patients with ankyloglossia. 34 Bottle feeding and eating solid foods do not pose problems for children with ankyloglossia. With age and usage, progressive stimulation and thinning of the frenulum occurs, and it is spontaneously able to extend; this may account for the general perception that ankyloglossia is more frequently seen in young children [4]. 
The relation between ankyloglossia and speech function is not yet clearly defined, although speech problems historically can be manifested as one of the symptoms of ankyloglossia; however, some literature denies that any such relationship exists. Ankyloglossia is rarely associated with speech problems, but in some individuals, ankyloglossia contributes to difficulties or errors in articulation with the rate and range of articulation mainly affecting the letters that require movement of the tip of the tongue, such as T, D, Z, S, TH, and L. To permit clear speech, an alternative tongue placement for sounds that require tongue-tip elevation and limit mouth opening when talking is a compensatory mechanism used by children with ankyloglossia [4].

In dentistry, diastema between the lower anterior teeth may be found in children with ankyloglossia as a result of pressure from the frenulum. On the other hand, ankyloglossia may cause difficulty in the process of intraoral cleaning, such as licking of the lips or removal of food residue in the mouth, a sense of discomfort, sores under the tongue, and difficulties in the use of dentures later in life [4].

Effects of ankyloglossia are not only experienced by children but also by their mothers, who can experience pain when the child latches on; get a cut; bleeding, flattened, painful, or wrinkled nipples; and failure to bond with the infant, causing depressed or plugged, ducts, engorgement, mastitis, or thrush [8].

\subsection{Pathophysiology of ankyloglossia}

The pathogenesis of ankyloglossia is still unclear. Some studies characterize ankyloglossia as an autosomal dominant inherited trait with incomplete penetrance, which can usually be seen in carrier females and sometimes in men [3,9]. The failure of genetically susceptible individuals to exhibit a trait is called incomplete penetrance $[10,11]$. However, other studies have proposed that ankyloglossia is inherited as an X-linked recessive condition and that the mothers of female patients were carriers [5].

Ankyloglossia is usually an isolated condition in normal childhood, but it can be a clinical feature of certain syndromes, e.g., X-linked Van Der Woude syndrome or cleft palate (syndromic and non-syndromic) [3,9]. Ankyloglossia can also be found in children with Opitz syndrome and orodigitofacial syndrome [4].

\subsection{Treatment}

Surgical procedures such as frenuloplasty, frenotomoy, and frenectomy or other modern techniques such as $\mathrm{Co} 2$ laser are the treatment options for ankyloglossia [5].

\section{Genetic}

\subsection{Definition}

DNA is a genetic material that consists two main molecular features: a complementary sequence of paired bases and a double helical polymer structure. Two polynu- 
cleotide chains wound around each other in the form of a double helix are characteristic of DNA (Fig 1). Each nucleotide consists of a phosphate joined to a sugar, known as 2'-deoxyribose, to which a base is attached [ ${ }^{12]}$.

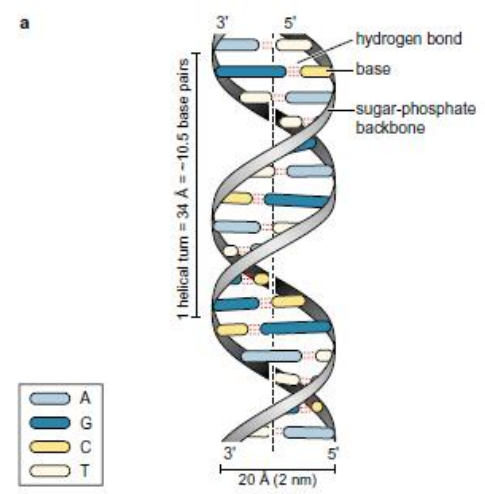

Fig 1. Schematic model of the double helix (source: Watson J. The Structures of DNA and RNA. In: Molecular Biology of Genes; 2004:1-33.)

Invariable changes in the DNA sequences that make up genes are called gene mutations, and they lead to sequences (wild type) that differ from those commonly found. Single DNA building blocks (base pairs) to huge segments of chromosomes, which can include multiple genes, can be affected by mutations. For a change to be recognized, there must be a "gold standard", compared to which the variant shows a difference [13]. Gene mutations can be classified into hereditary mutations (inherited from parents, also called germline mutations) and acquired (or somatic) mutations (caused by environmental factors such as UV) [14].

Mutations are sometimes classified by the size of the altered DNA sequence and other times by the functional effects of the mutation on gene expression. Mutation can occur at three different levels [13].

a. mutations that leave chromosomes intact but change the number of chromosomes in the cells (chromosome mutations)

b. mutations that change only a portion of a chromosome and might involve a change in the copy number of a sub-chromosomal segment or a structural rearrangement involving parts of one or more chromosomes (regional or sub-chromosomal)

c. alterations of the sequences of DNA, involving the substitution, deletion, or insertion mutations) of DNA, ranging from a single nucleotide up to an arbitrarily set limit of approximately $100 \mathrm{~kb}$ (gene or DNA mutations)

There are several types of mutation, the first being nucleotide substitution, which is divided into missense and nonsense mutations. A missense mutation occurs when a single nucleotide substitution (or point mutation) in a gene sequence, for example, in a patient with achondroplasia, alters the code in a triplet of nucleotide bases and causes a nonsynonymous amino acid change. Although not all missense mutations lead to an observable change in the function of the protein, the resulting protein may 
fail to work properly, may be unstable and thus rapidly degraded, or may fail to localize in its proper intracellular position [13].

Nonsense mutations are point mutations in a DNA sequence causing replacement of the normal codon for an amino acid by one of the three termination or stop codons. Translation of messenger RNA (mRNA) ceases when a termination codon is reached. There are two consequences of premature termination mutations. First, the mRNA carrying a premature mutation is often targeted for rapid degradation, and no translation is possible. Second, even if the mRNA is stable enough to be translated, the truncated protein is usually so unstable that it is rapidly degraded within the cell [13].

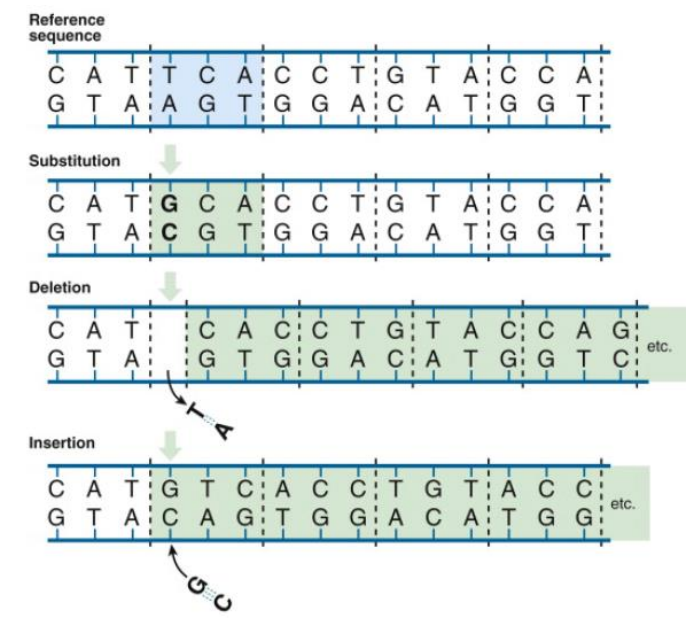

Fig 2. Types of mutations (source: Nussbaum RL, Cm RRM, Frs C, Willard HF. Mutation and Polymorphism. In: Human Genetic Diversity. Elsevier; 2017:43-56.)

\subsection{Genetic changes in the case of tongue tie and pathogenesis of tongue tie} 3.2.1 Isolated tongue tie

Little is known about the main pathogenesis of ankyloglossia/tongue tie. The literature reports two genes associated with this condition. The first is TBX22, which is responsible for causing ankyloglossia that does not accompany a syndrome (isolated feature). TBX22 is a "factor gene transcription T-box"; its cytogenetic location is on human chromosome Xq21.1 (Fig 3). Mutation in this gene can cause $\mathrm{X}$-linked cleft palate and ankyloglossia. The second gene is Lgr5, "an orphan G protein-coupled receptor gene" related to lethality in newborn mice. In these mice, targeted gene deletion was carried out, and the resulting phenotype was ankyloglossia. The cytogenetic location of the LGR5 gene is on human chromosome 12q21.1 (Fig 4) [2,3].

Ankyloglossia may be present with or without cleft lip and palate. The TBx22 (T-box transcription factor) gene is active in the process of shelf palate and base mouth development. Confirmation of the relationship between mutation in the TBX22 gene with cleft palate with ankyloglossia (CPX), whether as an isolated 
defect (ankyloglossia or cleft palate) or a combination of cleft lip and palate with ankyloglossia, have been reported recently by several studies [2,9].

Kantapura (2011) examined whether ankyloglossia with or without craniofacial abnormalities was caused by a gene mutation in TBX22 and reported five missense gene mutations. Two patients with mutations R120Q and P389Q were identified as having isolated ankyloglossia. A gene mutation in TBX22 was reported to cause ankyloglossia in some patients in a Thai family. A 9-year-old girl in Thailand was reported to be heterozygous for two mutations consisting of a missense in exon 8 $(1166 \mathrm{C}>\mathrm{A})$ and a missense in exon $3(359 \mathrm{G}>\mathrm{A})$ with P389Q. The amino acid substitution R120Q was revealed in children aged 3 years in Thailand (Fig 3). Another study conducted Kantapura indicated that the gene mutation $11133 \mathrm{G}>\mathrm{A}$, resulting in the amino acid substitution E187K, in the Tbx22 gene can cause ankyloglossia (Fig 5), but is not a main factor in ankyloglossia in the Finnish population. Eleven types of missense mutation associated with the classic CPX phenotype at the location of R120W reportedly occurred in a Tunisian Family [15]

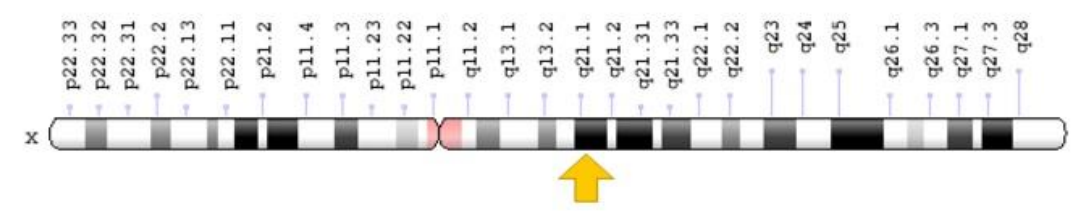

Fig 3: Cytogenic location of TBX22 genes (source: Weizmann Institute of Science. Human Gene Database TBX22. LifeMap Sciences. http://www.genecards.org/cgibin/carddisp.pl?gene $=$ TBX22.)

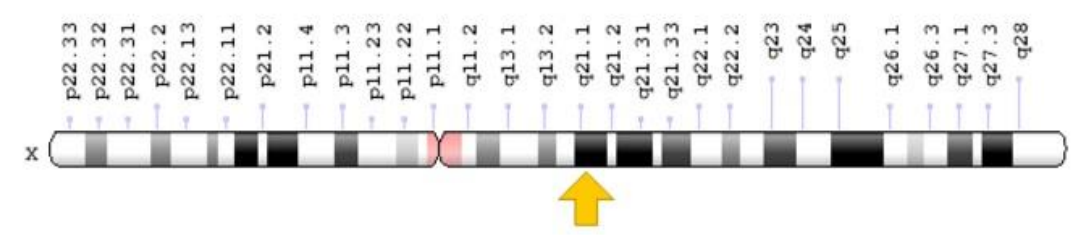

Fig 4: Cytogenic location of LGR5 genes (source: Weizmann Institute of Science. Human Gene Database LGR5. LifeMap Sciences. http://www.genecards.org/cgibin/carddisp.pl?gene=LGR5) 


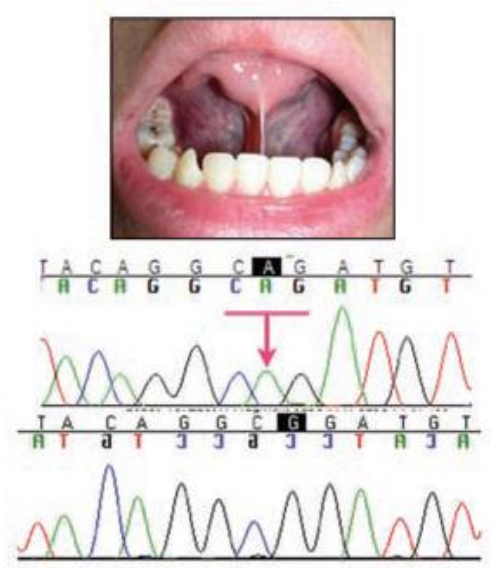

Fig 5: The missense mutation $359 \mathrm{G}>\mathrm{A}(\mathrm{R} 120 \mathrm{Q})$ was revealed in patients with isolated ankyloglossia (source: Kaewkhampa A, Jotikasthira D, Malaivijitnond S. Case Reports TBX22 Mutation Associated with Cleft Lip/Palate, Hypodontia, and Limb Anomaly. Cleft Palate-Craniofacial J. 2012;49(2):240-243.)

Lgr5 encodes a 907-amino-acid protein, the gene locations in individual mice of which were on chromosome $10 \mathrm{qD} 2$, whereas in humans, this gene is located on chromosome 12q21.1. Lgr5, which spans 144809 bp of the genome, is organized into 18 coding exons. The mice die within 24 hours due to defective sucking caused by deletion of the Lgr5 gene. Histologic analysis suggested immunostaining of the Lgr5 gene in epithelial cells of the tongue and mandible, and fusion of the tongue to the base of the mouth appeared in the baby mutant mice, whereas the embryo appeared to have the wild type, showing that the role of Lgr5 is in the signal pathway involved in proper tongue development $[2,18]$

However, a study conducted by Acevedo (2010) excluded mutase in the coding region of the Lgr5 gene as a causative aspect in families with ankyloglossia defects with tooth number anomalies. Yet, they did not rule out the possibility that mutase in the promotor region or within introns may affect expression of the Lgr5 gene or may be responsible for the syndrome. Lgr5 remains an attractive candidate for investigation in a large cohort study of patients with ankyloglossia $[2,18]$.

\subsubsection{Tongue tie related to cleft palate}

Generally, tongue tie can also be present accompanied by a cleft lip and palate. Several types of mutation in the TBX22 gene, such as missense, splice site, and nonsense mutations, were associated with this condition in several families (Clave, 2001). 


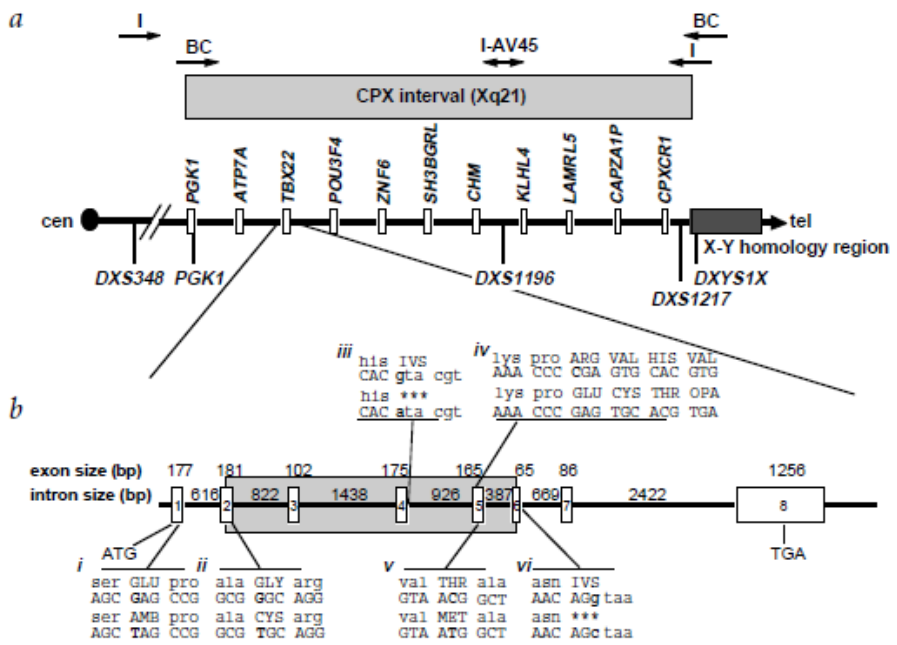

Fig 6: Genetic code in a patient with tongue tie accompanied by cleft lip and palate.

Candidate genes identified in the CPx interval in Xq21 (source: Jaenisch R, Bird A. Epigenetic regulation of gene expression: how the genome integrates intrinsic and environmental signals. Nat Publ Gr. 2003;33(March):245-254. doi:10.1038/ng1089.)

\section{Epigenetics}

\subsection{Definition of epigenetics}

Gene expression in cells is regulated on three different levels: first, the genetic code determines which proteins can potentially be made by an organism; second, the epigenetic code modifies chromatin so that transcription is either stimulated or suppressed; and third, transcription factors bind to initiate gene transcription [19].

In 1942, Waddington introduced the term epigenetics, which is derived from the Greek, "epigenesist". Initially, the word epigenetics described genetic effects occurring during the process of development. Today, epigenetics is the study of changes in the functions of genes, which can be mitotically and/or meiotically derived and do not affect the DNA sequence. The epigenetic code should be modified to include changes in the expression of genes necessary for development and differentiation. Epigenetic modification is a normal and natural event that arises during development and cell proliferation, but it can also relate to several factors, for example, the environment, state of the disease, or age. In this way, cells can adapt to the changing environment to stabilize the expression patterns of genes [19,20].

Three systems (DNA methylation, histone modification, and non-coding RNA gene silencing) are considered to initiate and maintain epigenetic changes [21]. Epigenetic modifications can be placed directly on the DNA base cytosine or on the protruding tails of histones. They can affect gene transcription both directly and 
indirectly. Direct effects are caused by changes in the packing of chromatin, which alter the accessibility of transcription factors, whereas indirect effects are mediated by the recruitment of effector molecules [19].

There are five histone proteins, namely histones $\mathrm{H} 1, \mathrm{H} 2 \mathrm{~A}, \mathrm{H} 2 \mathrm{~B}, \mathrm{H} 3$, and $\mathrm{H} 4$. They keep DNA packaged inside the nucleus so that the positively charged amino acids of the histone tails can interact with the negatively charged phosphate backbone of DNA. If negatively charged epigenetic modifications are placed on histone tails, they induce a conformational change that loosens the chromatin structure and thus induces the formation of transcribed euchromatin. Other modifications tighten the packaging of DNA, which results in inactive heterochromatin. In addition, effector molecules are bound that lead to stabilization or inhibition of the transcription machinery. There are some modifications of histone tails, namely acetylation, methylation, phosphorylation (induced and removed rapidly after stress stimuli), ubiquitylation (addition of ubiquitin to lysines in histone tails), SUMOylation (which has the same structural features as ubiquitination but different functions in transcriptional control), glycosylation, biotinylation, citrullination, $\mathrm{N}$-formylation, crotonylation, propionylation, butyrylation, carbonylation, and adenosine diphosphate (ADP) ribosylation [22]. These modifications are not isolated but are interconnected and affect gene transcription in combination or sequentially. Although all these histone modifications are assumed to modulate transcriptional activity, they vary widely in stability and impact, for example, in their ability to initiate changes in gene expression [19].

SUMOylation, one of the histone modifications, is the addition of a small, ubiquitin-related modifier to lysines in the histone tail. SUMOylation is considered as a counter player to acetylation because it occurs more on acetylated lysines with attractive HDACs, leading to the removal of acetylation and the repression of gene transcription. Transcriptional repression can then be further reinforced by the binding of heterochromatin protein 1 (HP1). HP1 works together with HMTs to induce stable repression via histone methylation. SUMO is conjugated to lysines by several enzymes, e.g., E1 SUMO (activating enzymes), E2 SUMO (conjugating enzymes), and E3 SUMO (ligases) and removed by isopeptidases. 19 Modification by SUMOylation affects the control of the Tbx22 gene, the gene responsible for the oral cleft facial expression. SUMOylation in histone modification is also necessary in regulating gene transcription, chromosome segregation, nuclear integrity, and embryonic viability. Therefore, with a heavy workload, SUMOylation requires support to ensure a healthy pregnancy [23].

Lately, DNA methylation has been the most widely studied aspect of epigenetic modification. Previous study indicated that DNA methylation might have an important function in long-term memory. Due to their experiments, awareness in the medical field has increased as to the importance of an appropriate pattern of genome methylation because it has an important role in healthy cells and organs [24].

DNA methylation is a biological process in which methyl groups, organic functional groups using $\mathrm{CH} 3$, are added to DNA nucleotides. The methyl group can bind to the carbon atoms derived from cytosine or the nitrogen atoms in adenine bases in the chromosomal DNA molecule. Cytosine methylation is the only modification of endogenous DNA known to be present in mammals where enzymatic addition of 
the methyl group to the $5 \square$ position of cytosine is present. The majority of 5 'methylcytocine in mammalian DNA is present in the dinucleotide 4'-CpG-3 '. Methylation patterns in specific genomic regions can be inherited [24].

If an appropriate methylation pattern is established or maintained, disorders such as immune deficiency, mental retardation, or cancer can occur. Through the simultaneous destabilization of whole chromosomes and inappropriate silencing of growth regulating genes, the methylation defect can create a chaotic state in which cancer cells develop. Abnormal methylation is much more common than one would expect [24].

There is a causal relationship between folate deficiency, which induces DNA hypomethylation, and cancer. The presence of genetic factors associated with folate metabolism determines the level of genome methylation. Enzymes involved in the synthesis of the S-adenosylmethonine (SAM) methyl donor are encoded by the methylenetetrahydrofolate reductase (MTHFR) gene, and the presence of specific polymorphisms in the MTHFR gene can reduce enzyme activity. DNA methylation is directly related to RBC folate levels in subjects with reduced MTHFR activity. Abnormal chromosomal segregation is associated with DNA hypomethylation. The study reported that that the presence of the MTHFR polymorphism may be a risk factor for Down syndrome and maternal meiotic non-disjunction in children of young mothers, and it is also associated with an increased risk of vascular disease and neural tube defects and may modify cancer risk [24]. There are also interesting aspects of the epigenetics associated with mental retardation, immune disorders, neuropsychiatric disorders, and pediatric disorders [21].

In the field of dentistry, many opportunities for diagnosis, treatment plans, and prevention can be opened up by new studies on epigenetic regulation. The genome is one factor that plays an important role in the development of teeth and various oral diseases. Oral microbes also play an important role in general health conditions. There is currently a potential to develop patient epigenetic profiles of patients to provide more personalized care. In oral health, the potential for epigenetic research lies in inflammation and the immune response, which cause periodontitis and the development of malignancies. There is a possibility to suppress the body's response against oral disease manifestation to maintain dental implants and prevent tooth loss. The most important thing that we can do is early screening for oral problems that may occur to prevent or reduce their impact. Epigenetics offers many opportunities in dental care prevention to prevent dental disease in the future [21]. 


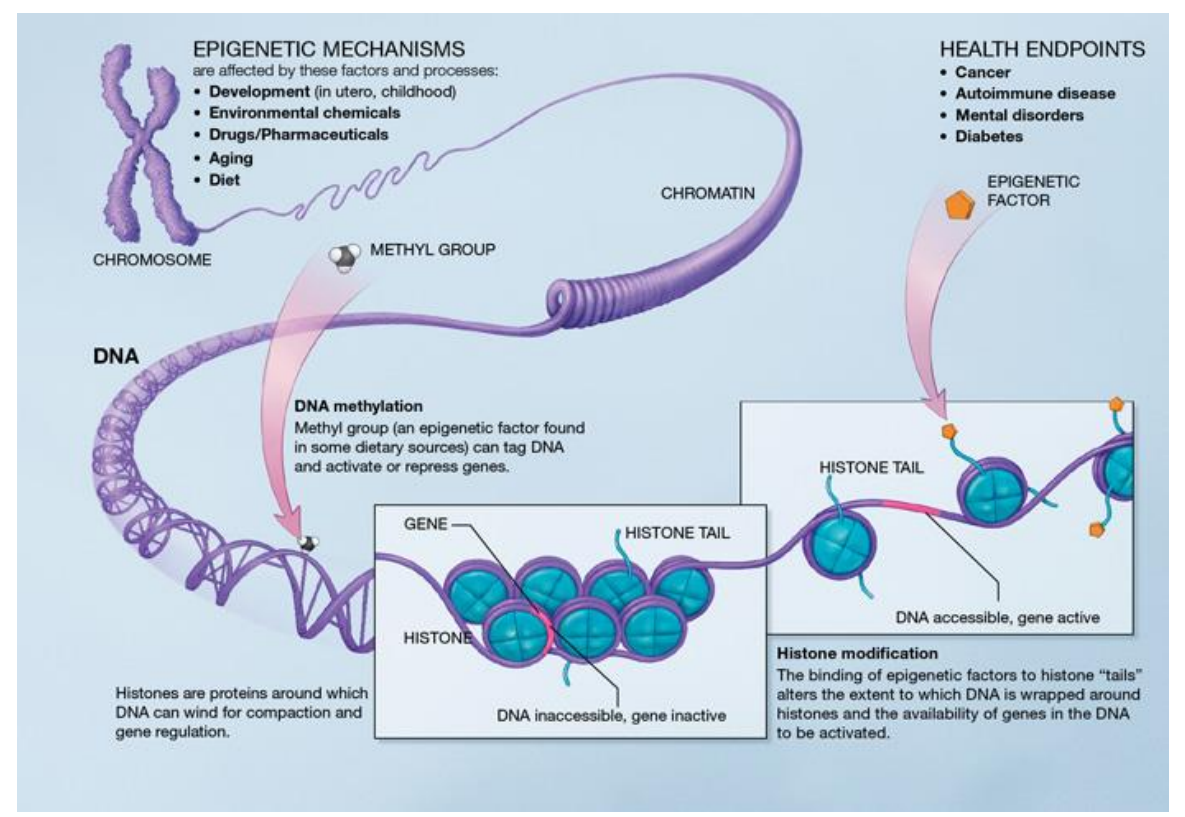

Fig 7: epigenetic mechanisms (source: Ospelt C, Gay S. Epigenetics in Rheumatology. ClinicalKey. 2016; 18:139-144.)

\subsection{Epigenetic modifications in ankyloglossia}

Besides mutation in the TBX22 gene, ankyloglossia is also associated with histone modification by SUMOylation. The presence of factors such as smoking, alcohol, lack of nutrients, hypoxia, viral infection, stress, etc. increase the risk of ankyloglossia; it is expected that reducing these risk factors can reduce the rate of occurrence of ankyloglossia [23].

One of the known factors contributing to SUMOylation is viral infection, which can be prevented by the presence of DNA methylation as immunity to the virus, because DNA methylation can suppress viral gene expression. It can be concluded that the presence of tongue tie is associated with the MTHFR gene, which is a regulator of methylation. As methylation is one of the biochemical reactions that are most important to our bodies in order to obtain the healthy function of DNA and overall health, insufficient methylation or an MTHFR gene mutation can lead to ankyloglossia [8].

The necessary steps to support methylation include obtaining adequate folate intake, limiting or reducing the consumption of foods which contain the polyphenol catechol, e.g., potatoes, sweet potatoes, or drinks such as coffee and tea, especially during the first trimester of pregnancy because they can inhibit DNMT-1 (the body's main methylator), and folate supplementation (in the form of folic acid or methyl folate) in early pregnancy and before conception [23]. 


\section{Conclusion}

Ankyloglossia, which is known as "tongue tie", is a congenital disorder mostly found in newborns. It is a condition in which the frenulum lingual hypertrophy is thicker and shorter than usual, thus limiting the mobility of the tip of the tongue, which is believed to interfere with breast feeding and speech, causing an oral motor disorder and social problems in the future. The prevalence of ankyloglossia is approximately $4 \%$ and is higher in men than women. The classification of ankyloglossia is still unclear.

Some studies report that ankyloglossia is an autosomal dominant disease with incomplete penetrance. Ankyloglossia may be present isolated or accompanied by cleft palate (CPX). The presentation of ankyloglossia is associated with nucleotide base substitution mutations in the gene TBX22. Another gene thought to be linked to ankyloglossia is the G-protein-coupled receptor (Lgr5) gene. The absence of this gene in mice can lead to ankyloglossia phenotypes and cause death within 24 hours due to feeding problems, but further studies are required in humans.

Current studies constantly find roles for epigenetics in many diseases and disorders in humans. Epigenetics is the study of changes in the functions of genes, which can be mitotically and/or meiotically derived and do not affect the DNA sequence. Epigenetic changes can have more damaging effects, such as cancer or malignancy. Epigenetic modifications that may be associated with ankyloglossia are DNA methylation and SUMOylation, which is one kind of histone modification.

If the cause and effect of different combinations of genes can be mapped and the gene state can be reverted to the wild type while the causative factor is removed, epigenetics can be used to slow the aging process, prevent obesity, cure diseases such as cancer, and prevent and treat dental problems.

\section{References}

1. Indonesia KK. Profil kesehatan Indonesia 2015. Jakarta; 2015.

2. Acevedo AC, da Fonseca JA, Grinham J, Doudney K, Gomes RR, de Paula LM, Stanier P. Autosomal-dominant ankyloglossia and tooth number anomalies. J Dent Res. 2010; 89(2):128-32.

3. Morowati S, Yasini M, Ranjbar R, Peivandi AA, Ghadami M. Familial ankyloglossia (tongue-tie): a case report. Acta Med Iran. 2010;48(2):123-4.

4. Lalakea ML, Messner AH. Ankyloglossia: does it matter? Pediatr. Clin. North Am. 2003;50(2):381-97.

5. Han SH, Kim MC, Choi YS, Lim JS, Han KT. A study on the genetic inheritance of ankyloglossia based on pedigree analysis. Arch Plast Surg. 2012;39(4):329.

6. Rajeev Agarwal. Ankyloglossia. 2015:1-29.

7. Hall DMB, Renfrew MJ. Tongue tie. 1988:1211-1215. www.archdischild.com.

8. Rittenour S. The Wellness Family. Park River Chiropractir Wellness.

9. Klockars T, Kyttänen S, Ellonen P. TBX22 and tongue-tie. Helsinki Univ Cent Hosp. 2011:378-379. doi:10.1597/11-114.

10. Department of health and human services. Inheriting Genetic Conditions. Genet Home Ref. 2017:8, 23.

11. Alper CA, Awdeh Z. Incomplete penetrance of MHC susceptibility genes: prospective analysis of polygenic MHC - determined traits. HLA. 2000;56(3):199-206. 
12. Watson J. The Structures of DNA and RNA. In: Molecular Biology of Genes.; 2004:133 .

13. Nussbaum RL, Cm RRM, Frs C, Willard HF. Mutation and Polymorphism. In: Human Genetic Diversity. Elsevier; 2017:43-56.

14. U.S national library of medicine. What is a gene mutation and how do mutations occur? Genetics Home Reference. https://ghr.nlm.nih.gov/primer/mutationsanddisorders/genemutation

15. Kaewkhampa A, Jotikasthira D, Malaivijitnond S, Kantaputra P. TBX22 mutation associated with cleft lip/palate, hypodontia, and limb anomaly. Cleft Palate Craniofac J. 2012;49(2):240-4.

16. Weizmann Institute of Science. Human Gene Database TBX22. LifeMap Sciences. http://www.genecards.org/cgi-bin/carddisp.pl?gene=TBX22.

17. Weizmann Institute of Science. Human Gene Database LGR5. LifeMap Sciences. http://www.genecards.org/cgi-bin/carddisp.pl?gene=LGR5.

18. Morita H, Mazerbourg S, Bouley DM, Luo CW, Kawamura K, Kuwabara Y, Baribault $\mathrm{H}$, Tian H, Hsueh AJ. Neonatal lethality of LGR5 null mice is associated with ankyloglossia and gastrointestinal distension. Mol Cell Biol 2004 15;24(22):9736-43.

19. Ospelt C, Gay S. Epigenetics in Rheumatology. ClinicalKey. 2016; 18:139-144.

20. Jaenisch R, Bird A. Epigenetic regulation of gene expression: how the genome integrates intrinsic and environmental signals. Nat gene. 2003; 33:245.

21. Mohsin AH, Barshaik S. Epigenetics in Dentistry: A Literature Review. J Clin Epigenetics. 2017 Jan 9;3(1).

22. Sadakierska-Chudy A, Filip M. A comprehensive view of the epigenetic landscape. Part II: Histone post-translational modification, nucleosome level, and chromatin regulation by ncRNAs. Neurotox Res. 2015;27(2):172-97.

23. Roseangove. The Intersection of Tongue Tie \& MTHFR. MTHFR and pregnancy. http://mthfr.net/the-intersection-of-tongue-tie-mthfr/2014/05/23/. Published 2014. Accessed March 20, 2017.

24. Costello JF, Plass C. Methylation matters. J Med Genet. 2001; 38:285-303. 